\title{
Chiral Kosterlitz-Thouless transition in the frustrated Heisenberg antiferromagnet on a pyrochlore slab
}

\author{
Hikaru Kawamura and Takuya Arimori \\ Department of Earth and Space Science, Faculty of Science, Osaka University, Toyonaka 560-0043, Japan
}

(November 9, 2018)

\begin{abstract}
Ordering of the geometrically frustrated two-dimensional Heisenberg antiferromagnet on a pyrochlore slab is studied by Monte Carlo simulations. In contrast to the kagomé Heisenberg antiferromagnet, the model exhibits locally non-coplanar spin structures at low temperatures, bearing nontrivial chiral degrees of freedom. Under certain conditions, the model exhibits a novel KosterlitzThouless-type transition at a finite temperature associated with these chiral degrees of freedom.
\end{abstract}

Magnetic ordering of geometrically frustrated antiferromagnets (AFs) has attracted interest of researchers in magnetism [1]3]. In geometrically frustrated AFs, spins usually sit on lattices made up of triangles or tetrahedra as elementary units, and interact antiferromagnetically with their neighboring spins. Intrinsic inability to simultaneously satisfy all antiferromagnetic nearest-neighbor interactions on a triangle or on a tetrahedron necessarily leads to macroscopic frustration. This makes the spin ordering on these lattices a highly nontrivial issue.

Recently, interest has been focused on the properties of the lattices consisting of corner-sharing triangles or tetrahedra, e.g., the 2D kagomé lattice and the 3D pyrochlore lattice [4 6]. Due to the looser coupling among the frustrating units, these systems often remain paramagnetic down to low temperatures. Experimentally, however, many of the geometrically frustrated magnets regarded as typical kagomé or pyrochlore AFs exhibit a phase transition at a low but finite temperature, quite often a spin-glass (SG)-like freezing transition [4 6].

One of the best studied geometrically frustrated AFs is the $S=3 / 2$ Heisenberg kagomé AF, SrCrGaO (SCGO) [4. 5]. Experimentally, this material exhibits a SG-like transition at a finite temperature $T=T_{f}$ as in many other geometrically frustrated AFs, although $T_{f}$ is considerably lower than the Curie-Weiss temperature of this material due to the strong geometrical frustration [7, 8 . In spite of extensive experimental and theoretical efforts, the true nature of this SG-like transition of SCGO has remained elusive. Although SCGO has been regarded for some time as a typical model compound of the $2 \mathrm{D}$ kagomé AF, Monte Carlo (MC) simulations performed for the antiferromagnetic Heisenberg model on the 2D kagomé lattice failed to reproduce the SG-like transition as experimentally observed in SCGO [9,10], suggesting that the modeling of SCGO as the kagomé AF might be inadequate in capturing some essential aspects of this material. In fact, the underlying lattice structure of SCGO is not of a pure (single-layer) kagomé lattice, but rather, of a kagomé sandwich, or a "pyrochlore slab" 4, 4, 7, 7,8]. The structure of the lattice is illustrated in Fig.1: It consists of two 2D kagomé layers which sandwiches the sparse triangular layer in between. Note that this lattice is obtained by slicing the $3 \mathrm{D}$ pyrochlore lattice along the (111) direction into the slab geometry. Inelastic neutron- scattering measurements have indicated that the neighboring slabs are magnetically well separated along the $c$-axis 11. Hence, in modeling SCGO, one may safely neglect the inter-slab interaction and consider the $2 \mathrm{D}$ Heisenberg model on a pyrochlore-slab lattice.
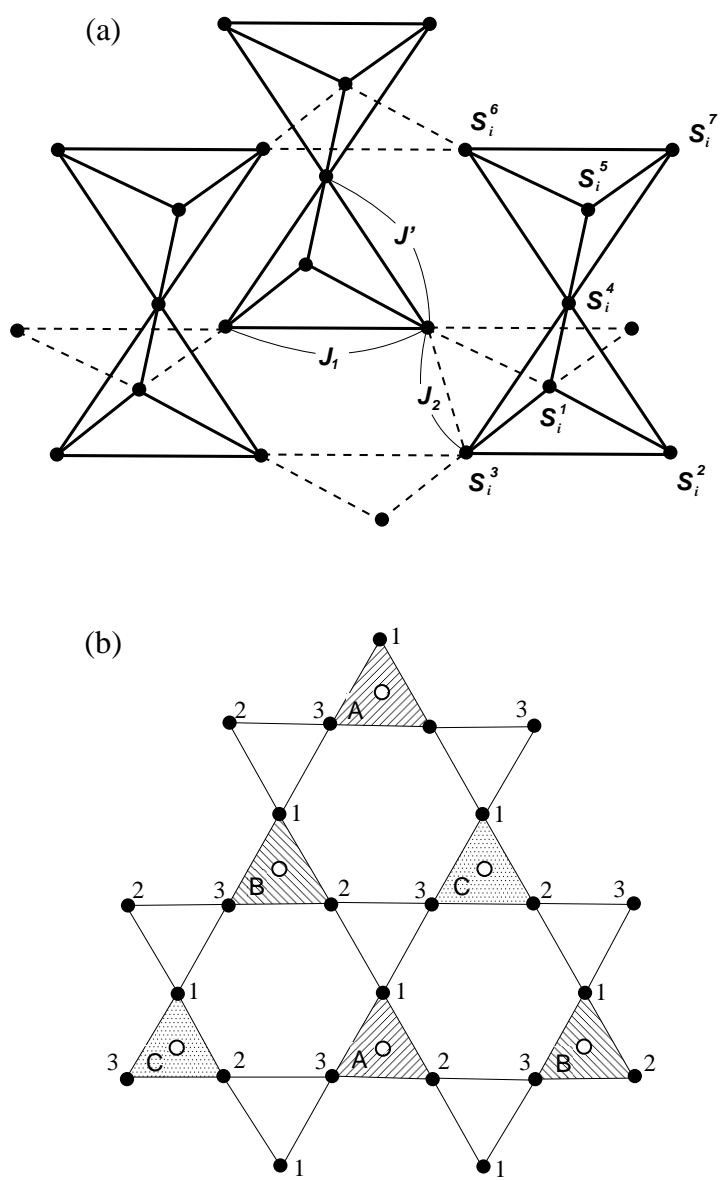

FIG. 1. A pyrochlore-slab lattice. (a) The unit cell of the lattice consists of two corner-sharing tetrahedra illustrated by the solid lines: (b) The lower kagomé layer consisting of the sites 1,2 and 3, denoted by the solid circle. The open circle denotes an apical site of tetrahedron (site 4). The upward triangles, shaded in the figure, correspond to the bottom planes of tetrahedra, and are grouped into three types A, B and $\mathrm{C}$, each forming the three inter-penetrating triangular sublattices. 
The purpose of the present Letter is to study the ordering properties of the antiferromagnetic classical Heisenberg model on a pyrochlore slab by means of MC simulations, and to examine whether some new features which are different from those of the well-studied pure kagomé Heisenberg AF would arise, possibly due to the tetrahedron-based structure of this lattice. In particular, we pay attention to the possible "chiral" properties of the model. "Chirality" is a multi-spin quantity representing the sense or handedness of the local non-coplanar spin structures induced by spin frustration. It is defined for three neighboring Heisenberg spins as a pseudo-scalar, $\chi=\vec{S}_{1} \cdot \vec{S}_{2} \times \vec{S}_{3}$, so as to give a nonzero value if the three spins make non-coplanar configurations but vanish otherwise. In the case of the pure kagomé Heisenberg AF, it has been known that the spin structure selected at low temperatures is a coplanar one with the vanishing chirality [9, 10]. In sharp contrast to this, we show in the case of the pyrochlore-slab Heisenberg AF that the spin structure stabilized at low temperatures is a non-coplanar one sustaining the nontrivial chirality. These nontrivial chiral degrees of freedom exhibit a novel thermodynamic phase transition at a finite temperature without accompanying the order of Heisenberg spins.

The unit cell of the pyrochlore-slab lattice may be taken as two corner-sharing tetrahedra containing seven sites, as illustrated by the solid lines in Fig.1(a). These unit cells containing seven sites, each numbered from 1 to 7 , are arranged forming the $2 \mathrm{D}$ triangular lattice of spacing $2 d, d$ being the lattice constant of the kagomé layers. In Fig.1(b), we show the lower kagomé layer consisting of the sites 1, 2 and 3. Note that the upward triangle in Fig.1(b) corresponds to the bottom plane of tetrahedron, while the downward triangle is not a part of any tetrahedron. These upward triangles are further grouped into three types, $A, B$ and $C$, each forming triangular sublattices of spacing $2 \sqrt{3} d$.

We consider the classical Heisenberg Hamiltonian,

$$
\mathcal{H}=\sum_{\langle i j\rangle} J_{i j} \vec{S}_{i} \cdot \vec{S}_{j}+J^{\prime} \sum_{\langle m n\rangle} \vec{S}_{m} \cdot \vec{S}_{n}
$$

where the antiferromagnetic interaction $J_{i j}$ on the kagomé layers is assumed to work between the nearestneighbor (nn) and the next-nearest-neighbor (nnn) pairs, with $J_{i j}=J_{1}$ and $J_{2}$, respectively, while $J^{\prime}$ is the antiferromagnetic nn interaction between the kagomé layers and the triangular layer. The variable $\vec{S}_{i}$ is a threecomponent unit vector at the $i$-th site. While we have made simulations for several choices of $J_{2} / J_{1}$ and $J^{\prime} / J_{1}$, we report here on our results for the case of antiferromagnetic nnn coupling $J_{2}>0$, because our model turns out to exhibit most interesting transition behavior in this case.

Since the present model is highly frustrated showing very slow relaxation at low temperatures, we combine the heat-bath MC method with the temperature-exchange technique to facilitate efficient thermalization [12]. Simulations are made for a pyrochlore slab with $N=7 \times N_{s}$ $\left(N_{s}=L \times L\right)$ spins with $L=6,12,18,24$ and 30. Periodic boundary conditions are employed.

Now, we present our Monte Carlo results for the particular case of $J_{2} / J_{1}=0.5$ and $J^{\prime}=J_{1}$. In Figs.2(a) and (b), we show the specific heat and the uniform susceptibility, respectively. The specific-heat data exhibit double peaks, at $T=T_{p 1} \simeq 0.29 J_{1}$ and at $T=T_{p 2} \simeq 0.09 J_{1}$. Their size dependence reveals that the peak heights eventually saturate with $L$, suggesting that both peaks are non-divergent ones. Meanwhile, the susceptibility data exhibit a clear cusp-like anomaly at $T / J_{1} \simeq 0.085$ close to the lower specific-heat peak.
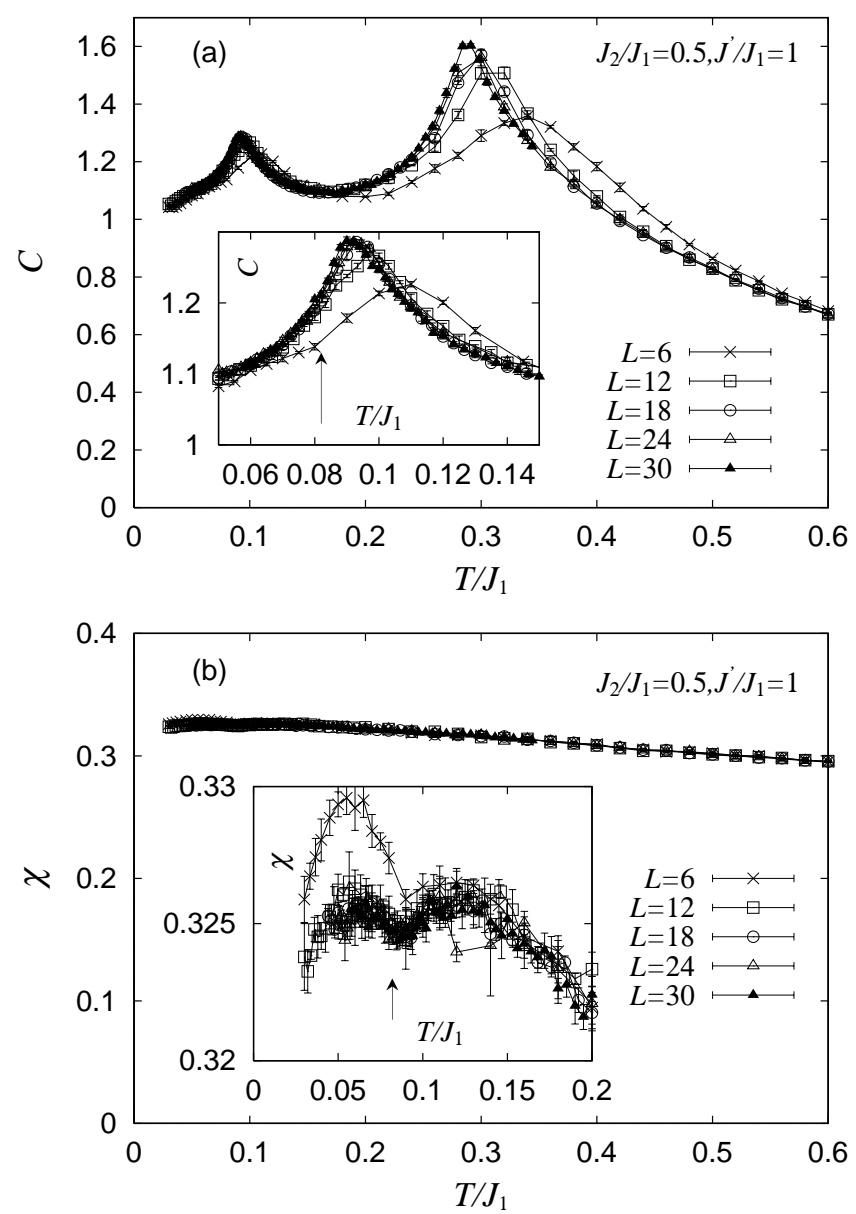

FIG. 2. The temperature and size dependence of (a) the specific heat and (b) the uniform susceptibility, per spin. The insets are magnified views of the low-temperature region. The arrow indicates the transition point.

One generally expects that, in a fully isotropic Heisneberg model in 2D like our model, there should be no long-range order (LRO) of Heisenberg spins, nor a finitetemperature transition occurring in the spin sector. We have confirmed this expectation by calculating various 
order parameters and Binder ratios associated with the spin order, e.g., the so-called $q=0$ and $\sqrt{3} \times \sqrt{3}$ orders on the kagomé layer [9, 10]. We also find that the higher specific-heat peak is correlated with the development of the $q=0$ short-range order of Heisenberg spins on the kagomé layers. In the present model with the antiferromagnetic $J_{2}$, the $q=0$ mode is selected over the $\sqrt{3} \times \sqrt{3}$ mode, in contrast to the pure kagomé Heisenberg AF where the $\sqrt{3} \times \sqrt{3}$ mode is selected [9, 10].
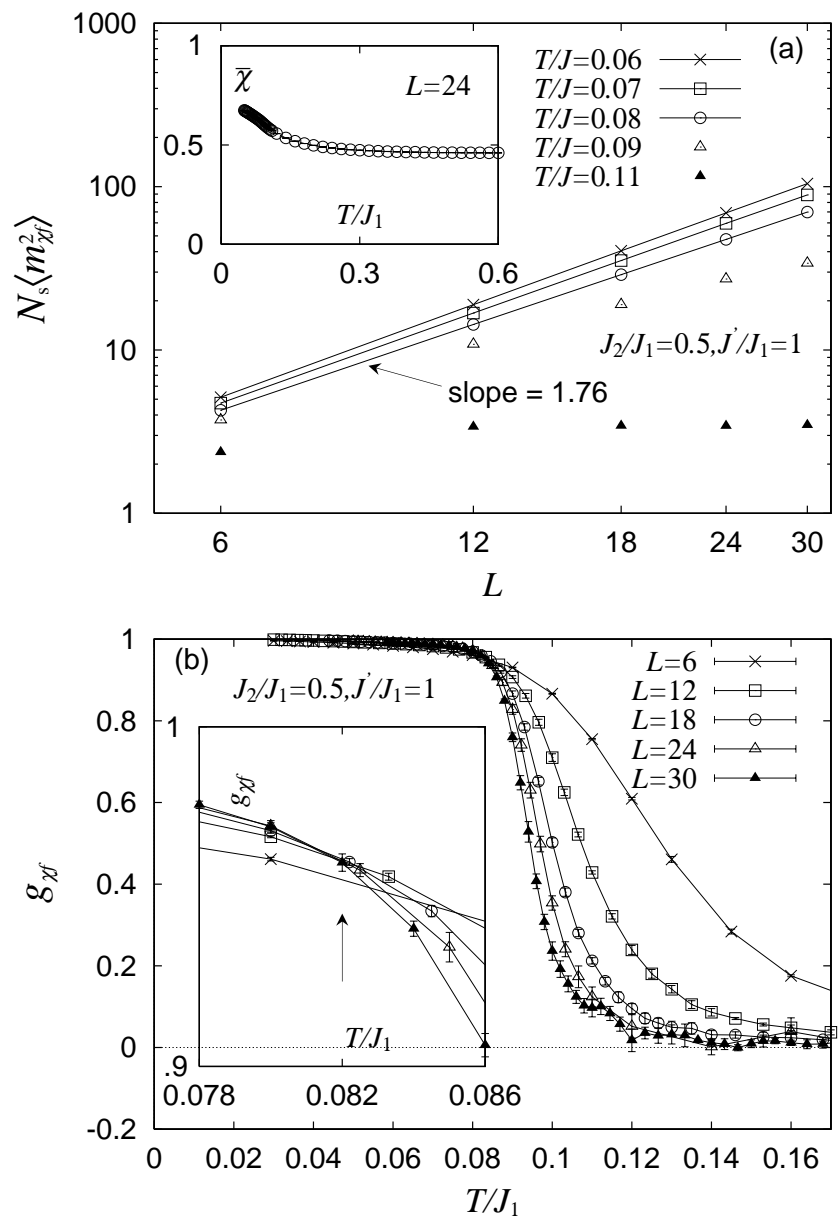

FIG. 3. (a) Log-log plot of the $L$-dependence of the chiral susceptibility associated with the $\sqrt{3} \times \sqrt{3}$ ordering at several temperatures. The inset represents the temperature dependence of the mean local amplitude of the chirality for $L=24$. The $L$-dependence of the data is negligible here, much smaller than the size of the symbols. (b) The temperature dependence of the chiral Binder ratio for various $L$. The inset is a magnified view of the transition region. The arrow indicates the transition point.

The chirality-related quantities are shown in Fig.3. We define here the local chirality, $\chi_{i}$, for the three spins 1 , 2 and 3 located at each upward triangle on the lower kagomé layer. In order to detect the local non-coplanarity of the spin structures, we compute the mean local amplitude of the chirality by $\bar{\chi}^{2}=\left(1 / N_{\mathrm{s}}\right) \sum_{i}\left\langle\chi_{i}^{2}\right\rangle$, where the summation is taken over all upward triangles and $\langle\cdots\rangle$ represents the thermal average. The data shown in the inset of Fig.3(a) indicate that in the $T \rightarrow 0$ limit $\bar{\chi}$ tends to a nonzero value, meaning that the spins belonging to tetrahedron form locally non-coplaner structures sustaining nontrivial chirality. The appearance of the nontrivial chiral degrees of freedom in the present model is in sharp contrast to the behavior of the pure kagomé Heisenberg AF.

In order to detect the possible ordering of these chiralities, we calculate the ferrimagnetic (or staggered) chiral order parameter $m_{\chi f}$, defined by

$$
\begin{gathered}
m_{\chi f}^{2}=\left(m_{\chi}^{A}\right)^{2}+\left(m_{\chi}^{B}\right)^{2}+\left(m_{\chi}^{C}\right)^{2} \\
-m_{\chi}^{A} m_{\chi}^{B}-m_{\chi}^{B} m_{\chi}^{C}-m_{\chi}^{C} m_{\chi}^{A}, \\
m_{\chi}^{A}=\frac{3}{N_{s}} \sum_{i \in A} \chi_{i}, \quad m_{\chi}^{B}=\frac{3}{N_{s}} \sum_{i \in B} \chi_{i}, \quad m_{\chi}^{C}=\frac{3}{N_{s}} \sum_{i \in C} \chi_{i} .
\end{gathered}
$$

This quantity gives a nonzero value if the chirality exhibits a ferrimagnetic order with $\sqrt{3} \times \sqrt{3}$ periodicity on three sublattices $A, B$ and $C$ in Fig.1(b). The associated chiral Binder ratio may be defined by

$$
g_{\chi f}=2-\frac{\left\langle m_{\chi f}^{4}\right\rangle}{\left\langle m_{\chi f}^{2}\right\rangle^{2}} .
$$

In Fig.3(b), we show the calculated chiral Binder ratio. For smaller $L, g_{\chi f}$ for various $L$ tend to cross at $T=T_{\mathrm{c}} \simeq 0.082 J_{1}$, but for larger $L$ they tend to merge at $T \leq T_{c}$, signaling the occurrence of a phase transition of the chirality. The estimated transition temperature $T_{\mathrm{c}} / J_{1}=0.082(2)$ is in rough agreement with the susceptibility-cusp temperautre estimated above, and is slightly below the lower specific-heat peak temperature $T_{p 2}$. In fact, there is no apprecialbe anomaly in the specific heat just at $T=T_{\mathrm{c}}$ : See the inset of Fig.2(a). A merging behavior of the Binder ratio, without the discernible specific-heat anomaly just at $T_{c}$ but with a nondivergent peak slightly above $T_{c}$, suggests that the observed chirality transition might be of the KosterlitzThouless (KT)-type.

In Fig.3(a), we show on a log-log plot the $L$-dependence of the chiral susceptibility $N_{s}\left\langle m_{\chi f}^{2}\right\rangle$ associated with the ferrimagnetic chiral order. While the data at higher temperatures exhibit the behaviors characteristic of the disordered phase, bending down toward some finite values, those at $T \leq T_{c}$ lie on straight lines, exhibiting the behavior expected for the KT-like phase with algebraicallydecaying correlations. The estimated slope of the plots, which should be equal to $2-\eta$ with $\eta$ being the criticalpoint decay exponent, turns out to be $\eta=0.24 \pm 0.01$ at $T=T_{c}$ and gradually decreases with decreasing temperature below $T_{c}$. This again indicates that the observed chiral transition at $T=T_{c}$ is the KT-type transition. 
Since the chirality here is an Ising like quantity sitting on the triangualr lattice, there is a close similarity between the chirality ordering of the present model and the ordering of the 2D Ising model on the triangular lattice. Indeed, when the triangular Ising model possesses the antiferromagnetic $\mathrm{nn}$ interaction and ferromagnetic nnn interation, the model is known to exhibit a $\sqrt{3} \times \sqrt{3}$ order via the KT-type transition characterized by the exponent $\eta=1 / 4$ 13 16]. Since the chirality ordering of the present model with $J_{2}>0$ is essntially the staggered one, it would be no surprise that the chirality ordering here is of the KT-type. To the authors' knowledge, our present finding is the first case of chiral KT transition without accompanying the spin order.

It should be noticed that the triangular Ising AF with the ferromagnetic nnn interaction exhibits another phase transition with further decreasing temperature, into the low-temperature phase with a finite LRO. [13 16] The exponent $\eta$ at this second transition is believed to be $1 / 9$ 13, 16, 17. In view of this, we search for this second transition into the long-range-ordered state in our present model, but with negative result. Presumably, severe frustration inherent to the present model might hinder the onset of the true LRO.

Although we have so far considered the case where the inter-plane interaction $J^{\prime}$ is equal to the intra-plane $\mathrm{nn}$ interaction $J_{1}$, such equality is not expected in general in real experimental systems. In order to examine the possible effect of varying the inter-plane coupling, we also performed similar calculations for the $J^{\prime}=0.5 J_{1}$ case, to get qualitatively similar results as the $J^{\prime}=J_{1}$ case. We thus conclude that the $J^{\prime}$ value is irrevant to the chiral KT transition observed here. Further detailed results of our MC simulations, including the results for other choices of the nnn interaction $J_{2}$, will be published elsewhere [18]. For the cases of the vanishing and ferromagnetic nnn interaction $J_{2} \leq 0$, we find that, although the local spin structure is non-coplanar sustaining nontrivial chirality, the chiral KT transition does not take place there.

Finally, we wish to refer to the possible experimental implication of our present results. We emphasize that the present model is expected to capture essential geometrical ingredients of SCGO, and indeed, seems to accout for some of the experimental features, e.g., the occurrence of a finite-temperature phase transition above all, the cusplike anomaly observed in the susceptibility [7], or the existence of a broad specific-heat peak slightly above $T_{c}$ [7]. However, we mention that some other experimental features remain unexplained. For example, experimentally, the transition is characterized by the negative divergence of the nonlinear susceptibility and the onset of magnetic irreversibility, e.g., a notable difference between the fieldcooled and zero-field-cooled magnetizations [7]. We could not reproduce these features in our simulations.

Although further work is clearly required to fully account for the experimental observation on SCGO, our present finding of the chiral KT transition in the pyrochlore-slab Heisenberg AF might hopefully give a new perspective in the studies of SCGO, and geometrically frustrated AFs in general.

The numerical calculation was performed on the Hitachi SR8000 at the supercomputer center, ISSP, University of Tokyo.

[1] Magnetic Systems with Competing Interaction ed. H.T. Diep, World Scientific (1994).

[2] M. Collins and O.A. Petrenko, Can. J. Phys. 75, 605 (1997).

[3] H. Kawamura, J. Phys. Condes. Matter 10, 4707 (1998).

[4] A. P. Ramirez, Ann. Rev. Mater. Sci. 24, 453 (1994).

[5] P. Schiffer and A. P. Ramirez, Comments Cond. Mat. Phys. 18, 21 (1996).

[6] M.J. Harris and M.P. Zinkin, Int. J. Mod. Phys. B 10, 417 (1996).

[7] A. P. Ramirez, G. P. Espinosa and A. S. Cooper, Phys. Rev. Lett. 64, 2070 (1990); Phys. Rev. B 45, 2505 (1992).

[8] C. Broholm, G. Aeppli, G. P. Espinosa and A. S. Cooper, Phys. Rev. Lett. 65, 3173 (1990).

[9] J. T. Chalker, P. C. W. Holdsworth and E. F. Shender, Phys. Rev. Lett. 68, 855 (1992).

[10] J. N. Reimers and A. J. Berlinsky, Phys. Rev. B 48, 9539 (1993).

[11] S. -H. Lee, C.Broholm, G. Aeppli, T.G. Perring, B. Hessen and A. Taylor, Phys. Rev. Lett. 76, 4424 (1996).

[12] K. Hukushima and K. Nemoto, J. Phys. Soc. Jpn. 65, 1604 (1996).

[13] D. P. Landau, Phys. Rev. B 27, 5604 (1983).

[14] H. Takayama, K. Matsumoto, H. Kawahara and K. Wada, J. Phys. Soc. Jpn. 52, 2888 (1983).

[15] S. Fujiki, K. Shutoh, Y. Abe and S. Katsura, J. Phys. Soc. Jpn. 55, 3326 (1986).

[16] S. Miyashita, H. Kitatani and Y. Kanada, J. Phys. Soc. Jpn. 60, 1523 (1991).

[17] J. Jose, L. Kadanoff, S. Kirkpatrick and D. R. Nelson, Phys. Rev. B 16, 1217 (1977).

[18] T. Arimori and H. Kawamura, in preparation. 